AEI-2007-024

\title{
Dressing and Wrapping
}

\author{
A. V. Kotikov ${ }^{a, c}$, L. N. Lipatov ${ }^{b, c}$, A. ReJ ${ }^{d}$, M. Staudacher ${ }^{d}$ And \\ V. N. Velizhanin ${ }^{b, c}$ \\ a Bogoliubov Laboratory of Theoretical Physics \\ Joint Institute for Nuclear Research \\ 141980 Dubna, Russia \\ ${ }^{b}$ Theoretical Physics Department \\ Petersburg Nuclear Physics Institute \\ Orlova Roscha, Gatchina \\ 188300 St. Petersburg, Russia \\ c II. Institut für Theoretische Physik, Universität Hamburg \\ Luruper Chaussee 149, D-22761 Hamburg, Germany \\ ${ }^{d}$ Max-Planck-Institut für Gravitationsphysik \\ Albert-Einstein-Institut \\ Am Mühlenberg 1, D-14476 Potsdam, Germany
}

\begin{abstract}
We prove that the validity of the recently proposed dressed, asymptotic Bethe ansatz for the planar AdS/CFT system is indeed limited at weak coupling by operator wrapping effects. This is done by comparing the Bethe ansatz predictions for the four-loop anomalous dimension of finite-spin twist-two operators to BFKL constraints from high-energy scattering amplitudes in $\mathcal{N}=4$ gauge theory. We find disagreement, which means that the ansatz breaks down for length-two operators at four-loop order. Our method supplies precision tools for multiple all-loop tests of the veracity of any yet-to-be constructed set of exact spectral equations.
\end{abstract}




\section{Introduction and Verdict}

Two-dimensional integrable structures appeared for the first time in four-dimensional gauge field theories in the context of high-energy scattering in QCD. In a certain leading logarithmic approximation the scattering amplitudes of colorless particles are well described by the exchange of two effective particles, termed reggeized gluons. A compound of two of these particles is frequently called the pomeron. In the planar limit, the associated dynamics is governed by an integrable Hamiltonian 1 [1]. Shortly after, this Hamiltonian was identified as the direct sum of two commuting non-compact spin-zero Heisenberg magnets [2]. The length of this spin chain equals the number of reggeized gluons considered. Therefore the leading dynamics of the pomeron is described by a very short spin chain with two sites.

Some years later integrable spin chains also resurfaced in the analysis of planar one-loop anomalous dimensions of composite local "twist" operators in QCD [3, 4]. The integrable structures appearing, respectively, in the context of reggeization and of anomalous dimensions are frequently confused even though the considered physical phenomena are quite different. However, when focusing on a more symmetric relative of QCD, the $\mathcal{N}=4$ gauge theory, deep and surprising connections indeed link the respective integrable structures [3]. In the $\mathcal{N}=4$ case, the above "confusion" therefore actually expresses a profound insight.

The next step towards unravelling the exactly solvable structure of planar $\mathcal{N}=4$ gauge theory came through the discovery that not only the sector of quasi-partonic twist operators, but in fact the complete set of local composite operators is described at one-loop by an integrable $\mathfrak{p s u}(2,2 \mid 4)$ non-compact supermagnet [5]. Its spectrum is hence described by a nested Bethe ansatz. Much evidence was found that integrability is not destroyed by radiative corrections, and that the Bethe ansatz extends to higher loops [6, 7, 8]. This led to a set of higher loop Bethe equations [9], which were accurate to three-loop order, but nevertheless still incomplete at four loops and beyond, in two distinct ways.

- Firstly, the Bethe ansatz [9] contained an unknown dressing factor which was initially introduced in order to reconcile the integrable structures of gauge and string theory [10], linked through AdS/CFT, in certain long-operator limits [11]. The understanding of its necessity and structure was subsequently refined in a series of important papers [12. Its existence was finally indirectly proven through an impressive fieldtheoretic four-loop calculation [13], following a testing procedure proposed in [14]. Its precise form was written down in [15] contemporaneously with [13], and agrees quantitatively with the field theory computation to a very high precision [16]. A self-consistent derivation of the dressing phase from first principles is still lacking. Very recently, however, it was demonstrated that the proper convolution structure of the phase arises from a nested Bethe ansatz [17]. See also the comments in [18].

- Secondly, the Bethe ansatz [9] does not necessarily incorporate wrapping effects, as it is by construction [7] asymptotic. The point is that the all-loop dilatation operator

\footnotetext{
${ }^{1}$ It was shown in [1] that the Hamiltonian is a member of a set of mutually commuting charges generated by a monodromy matrix satisfying the Yang-Baxter equation. This Padua University preprint had been submitted to Physics Letters B and was rejected by the referee.
} 
of the gauge theory is long-range, i.e. the interactions link at $\ell$ loop orders $\ell+1$ neighboring sites on a lattice spanned by the partons. The definition of an S-matrix requires an asymptotic region (see [7] for a discussion). If the interaction range exceeds the size of the system the asymptotic region shrinks to zero and the Bethe ansatz might well break down. One can show [9] that in $\mathcal{N}=4$ this cannot happen up to three-loop order. A wrapping-induced breakdown might however occur at fourloop order for the shortest possible operators. Investigating this issue is the main purpose of this paper.

The shortest possible local composite operators in the $\mathcal{N}=4$ theory are the so-called twist-two operators. For a simple representative of these one starts from the protected half-BPS states $\operatorname{Tr} \mathcal{Z}^{2}$ and inserts $M$ covariant derivatives $\mathcal{D}$ :

$$
\operatorname{Tr}\left(\mathcal{Z} \mathcal{D}^{M} \mathcal{Z}\right)+\ldots
$$

In the spin chain picture this is a non-compact $\mathfrak{s l}(2)$ spin $=-\frac{1}{2}$ length-two Heisenberg magnet with $M$ magnonic excitations. The dots indicate the mixing of all states where the covariant derivatives may act on any of the two fields. For each even $M$ there is precisely one non-BPS state whose total scaling dimension is

$$
\Delta=2+M+\gamma(g), \quad \text { with } \quad \gamma(g)=\sum_{\ell=1}^{\infty} \gamma_{2 \ell} g^{2 \ell},
$$

where $\gamma(g)$ is the anomalous part of the dimension depending on the coupling constant

$$
g^{2}=\frac{\lambda}{16 \pi^{2}},
$$

and $\lambda=N g_{\mathrm{YM}}^{2}$ is the 't Hooft coupling constant. States with odd $M$ do not exist in the $\mathfrak{s l}(2)$ sector. The anomalous part $\gamma(g)$ of the dimension may be reliably computed to three-loop order $\mathcal{O}\left(g^{6}\right)$ by the asymptotic Bethe ansatz [7], see (2.1),(2.4) of chapter 2. The result agrees at two-loop order with the one obtained from an explicit field-theory calculation [19], and at three-loop order with a solid conjecture [20] extracted by the principle of maximum transcendentality [21] from a rigorous field theory calculation in QCD [22]. Closely related interesting properties of perturbative anomalous dimensions, following from certain generalized relations of Gribov and Lipatov, and of Drell, Levy and Yan, are discussed in [23].

In $\mathcal{N}=4$ theory the $\ell$-th loop anomalous dimension $\gamma_{2 \ell}(M)$ is expressed through a combination of harmonic sums of constant degree $2 \ell+1$. These are defined in (2.5) below. The relationship to the Balitsky-Fadin-Kuraev-Lipatov (BFKL) approach [24] for describing high energy scattering amplitudes in gauge theory appears upon analytically continuing the function $\gamma(g, M)$, and therefore the $\gamma_{2 \ell}(M)$, to general, complex values of $M$. In particular, one expects singularities at all negative integer values of $M$. The first in this series of singular points corresponds to the above mentioned pomeron at

$$
M=-1+\omega
$$


where $\omega$ should be considered small. Notice that in BFKL physics one more commonly uses the variable $j$ instead of $M$. These are related through $M=j-2$. Roughly speaking, in view of (1.1) we could say that the BFKL pomeron of $\mathcal{N}=4$ gauge theory is described by the non-local gauge-invariant operator

$$
\text { pomeron }=\operatorname{Tr}\left(\mathcal{Z D}^{-1+\omega} \mathcal{Z}\right) .
$$

The BFKL equation relates $\gamma(g)$ and $g$ in the vicinity of the point $M=-1$. To leading (LO) one-loop order it reads

$$
\frac{\omega}{-4 g^{2}}=\Psi\left(-\frac{\gamma}{2}\right)+\Psi\left(1+\frac{\gamma}{2}\right)-2 \Psi(1)
$$

where $\Psi(x)=\frac{d}{d x} \log \Gamma(x)$ is the logarithmic derivative of Euler's Gamma function. By expanding the $\Psi$-functions in infinite series it may be rewritten as

$$
\frac{\omega}{-4 g^{2}}=\frac{2}{\gamma}-2 \sum_{k=1}^{\infty}\left(\frac{\gamma}{2}\right)^{2 k} \zeta(2 k+1) \text {. }
$$

We are now ready to point out the crucial importance of the BFKL equation as a testing device for any, past or future, conjecture on the exact higher-loop spectrum of anomalous dimensions in the $\mathcal{N}=4$ model. The point is that even though (1.6) is only the one-loop approximation to the true, currently unknown relationship between the spin label $M=-1+\omega$ and the anomalous dimension $\gamma$, upon inversion of the power series (1.7) we get an all-loop prediction of the leading singular behavior of $\gamma$ as a function of the deviation from the singularity at $M=-1$ as $\omega \rightarrow 0$.

This inversion is easily performed to arbitrary orders of perturbation theory. To e.g. four-loop order one finds for the analytically continued anomalous dimension

$$
\gamma=2\left(\frac{-4 g^{2}}{\omega}\right)-0\left(\frac{-4 g^{2}}{\omega}\right)^{2}+0\left(\frac{-4 g^{2}}{\omega}\right)^{3}-4 \zeta(3)\left(\frac{-4 g^{2}}{\omega}\right)^{4} \pm \ldots
$$

This may now be compared to the result as obtained from the dressed asymptotic Bethe ansatz (ABA). In chapter 2 below we present its prediction for the four-loop dimension at arbitrary positive integer spin $M$, see table 1. After analytic continuation to negative values of the spin $M$, and expanding in $\omega$ around the pole at $M=-1$, see (1.4), we find

$$
\gamma^{\mathrm{ABA}}=2\left(\frac{-4 g^{2}}{\omega}\right)-0\left(\frac{-4 g^{2}}{\omega}\right)^{2}+0\left(\frac{-4 g^{2}}{\omega}\right)^{3}-2 \frac{\left(-4 g^{2}\right)^{4}}{\omega^{7}} \pm \ldots,
$$

where we have also restated the known results at less than four loops. One observes maximal violation of the BFKL prediction (1.8): The leading singularity in $\omega$ should be a pole of fourth order. Instead, we find a seventh order pole, which is the maximum order the analytic continuation of an harmonic sum of transcendentality degree seven can yield. This means that $\gamma^{\mathrm{ABA}}$ cannot be the correct anomalous dimension of finite spin $M$ twist-two operators in $\mathcal{N}=4$ gauge theory. We have thus established that the long-range asymptotic Bethe ansatz breaks down at four-loop order. 


\section{Four-Loop Twist-Two from Bethe Ansatz}

Let us now prove our claim. The twist $J=2$ operators (1.1) of interest to us sit in the $\mathfrak{s l}(2)$ sector of the full $\mathfrak{p s u}(2,2 \mid 4)$ magnet. The long-range asymptotic Bethe equations for twist- $J$ operators read in this sector [9, 15]

$$
\left(\frac{x_{k}^{+}}{x_{k}^{-}}\right)^{J}=\prod_{\substack{j=1 \\ j \neq k}}^{M} \frac{x_{k}^{-}-x_{j}^{+}}{x_{k}^{+}-x_{j}^{-}} \frac{1-g^{2} / x_{k}^{+} x_{j}^{-}}{1-g^{2} / x_{k}^{-} x_{j}^{+}} \exp \left(2 i \theta\left(u_{k}, u_{j}\right)\right), \quad \prod_{k=1}^{M} \frac{x_{k}^{+}}{x_{k}^{-}}=1 .
$$

These are $M$ equations for $k=1, \ldots, M$ Bethe roots $u_{k}$, with

$$
x_{k}^{ \pm}=x\left(u_{k}^{ \pm}\right), \quad u^{ \pm}=u \pm \frac{i}{2}, \quad x(u)=\frac{u}{2}\left(1+\sqrt{1-4 \frac{g^{2}}{u^{2}}}\right),
$$

and where the dressing phase $\theta$ is a rather intricate function conjectured in [15]. Here we will only need it to leading four-loop order, where it reads

$$
\theta\left(u_{k}, u_{j}\right)=4 \zeta(3) g^{6}\left(q_{2}\left(u_{k}\right) q_{3}\left(u_{j}\right)-q_{3}\left(u_{k}\right) q_{2}\left(u_{j}\right)\right)+\mathcal{O}\left(g^{8}\right),
$$

and where the $q_{r}(u)$ are the eigenvalues of the conserved magnon charges, see [9] for details on this formalism. Once the $M$ Bethe roots are determined from (2.1) for the state of interest, its asymptotic all-loop anomalous dimension is given by

$$
\gamma^{\mathrm{ABA}}(g)=2 g^{2} \sum_{k=1}^{M}\left(\frac{i}{x_{k}^{+}}-\frac{i}{x_{k}^{-}}\right) .
$$

The equations (2.1) can be solved recursively order by order in $g$ at arbitrary values of $M$ and $J$ once the one-loop solution for a given state is known.

It was checked in [7] up to relatively high values of spin, that this Bethe ansatz reproduces correctly the two- and three-loop anomalous dimensions of the twist $J=2$ operators, which are known in terms of nested harmonic sums as obtained in [19, 20]. However unfortunate, no analytical derivation is known at the time of writing. It would be extremely interesting to develop tools for solving this problem. Therefore, a priori it is even less clear how to extract the four-loop prediction from the above Bethe equations.

This technical problem can nevertheless be surmounted. Assuming the maximum transcendentality principle [21] at four-loop order one may derive the corresponding expression for the anomalous dimension by making an appropriate ansatz with unknown coefficients multiplying the nested harmonic sums, and subsequently fixing these constants. The latter is done by fitting to the exact anomalous dimensions for a sufficiently large list of specific values of $M$ as calculated from the Bethe ansatz.

Luckily, at one-loop the exact solution of the Baxter equation is known [14] and is given by a Hahn polynomial. Knowing the one-loop roots one can then expand equation (2.1) in the coupling constant $g$ order by order in perturbation theory. The equations for 


$$
\begin{aligned}
& \mathbf{4} \boldsymbol{S}_{-\mathbf{7}}+\mathbf{6} \boldsymbol{S}_{\boldsymbol{7}}+2\left(S_{-3,1,3}+S_{-3,2,2}+S_{-3,3,1}+S_{-2,4,1}\right)+3\left(-S_{-2,5}\right. \\
& \left.+S_{-2,3,-2}\right)+4\left(S_{-2,1,4}-S_{-2,-2,-2,1}-S_{-2,1,2,-2}-S_{-2,2,1,-2}-S_{1,-2,1,3}\right. \\
& \left.-S_{1,-2,2,2}-S_{1,-2,3,1}\right)+5\left(-S_{-3,4}+S_{-2,-2,-3}\right)+6\left(-S_{5,-2}\right. \\
& \left.+S_{1,-2,4}-S_{-2,-2,1,-2}-S_{1,-2,-2,-2}\right)+7\left(-S_{-2,-5}+S_{-3,-2,-2}\right. \\
& \left.+S_{-2,-3,-2}+S_{-2,-2,3}\right)+8\left(S_{-4,1,2}+S_{-4,2,1}-S_{-5,-2}-S_{-4,3}\right. \\
& \left.-S_{-2,1,-2,-2}+S_{1,-2,1,1,-2}\right)+9 S_{3,-2,-2}-10 S_{1,-2,2,-2}+11 S_{-3,2,-2} \\
& +12\left(-S_{-6,1}+S_{-2,2,-3}+S_{1,4,-2}+S_{4,-2,1}+S_{4,1,-2}-S_{-3,1,1,-2}-S_{-2,2,-2,1}\right. \\
& -S_{1,1,2,3}-S_{1,1,3,-2}-S_{1,1,3,2}-S_{1,2,1,3}-S_{1,2,2,-2}-S_{1,2,2,2}-S_{1,2,3,1}-S_{1,3,1,-2} \\
& -S_{1,3,1,2}-S_{1,3,2,1}-S_{2,-2,1,2}-S_{2,-2,2,1}-S_{2,1,1,3}-S_{2,1,2,-2}-S_{2,1,2,2} \\
& -S_{2,1,3,1}-S_{2,2,1,-2}-S_{2,2,1,2}-S_{2,2,2,1}-S_{2,3,1,1}-S_{3,1,1,-2}-S_{3,1,1,2}-S_{3,1,2,1} \\
& \left.-S_{3,2,1,1}\right)+13 S_{2,-2,3}-14 S_{2,-2,1,-2}+15\left(S_{2,3,-2}+S_{3,2,-2}\right) \\
& +16\left(S_{-4,1,-2}+S_{-2,1,-4}-S_{-2,-2,1,2}-S_{-2,-2,2,1}-S_{-2,1,-2,2}-S_{-2,1,1,-3}\right. \\
& -S_{1,-3,1,2}-S_{1,-3,2,1}-S_{1,-2,-2,2}-S_{2,-2,-2,1}+S_{-2,1,1,-2,1}+S_{1,1,-2,1,-2} \\
& \left.+S_{1,1,-2,1,2}+S_{1,1,-2,2,1}\right)-17 S_{-5,2}+18\left(-S_{4,-3}-S_{6,1}+S_{1,-3,3}\right) \\
& +20\left(-S_{1,-6}-S_{1,6}-S_{4,3}+S_{-5,1,1}+S_{-4,-2,1}+S_{-3,-2,2}+S_{-2,-4,1}\right. \\
& \left.+S_{-2,-3,2}+S_{1,3,3}+S_{3,1,3}+S_{3,3,1}-S_{1,1,-2,3}-S_{1,2,-2,-2}-S_{2,1,-2,-2}\right) \\
& -21 S_{3,4}+22\left(S_{1,-2,-4}+S_{2,2,3}+S_{2,3,2}+S_{3,-2,2}+S_{3,2,2}\right)+23\left(-S_{-3,-4}\right. \\
& \left.-S_{5,2}+S_{2,-2,-3}\right)+24\left(-S_{-4,-3}+S_{1,-4,-2}-S_{1,-3,1,-2}-S_{1,1,1,4}-S_{1,1,4,1}\right. \\
& -S_{1,3,-2,1}-S_{1,4,1,1}-S_{3,-2,1,1}-S_{3,1,-2,1}-S_{4,1,1,1}+S_{-2,-2,1,1,1}+S_{-2,1,-2,1,1} \\
& +S_{1,-2,-2,1,1}+S_{1,-2,1,-2,1}+S_{1,1,-2,-2,1}+S_{1,1,1,-2,-2}+S_{1,1,2,-2,1}+S_{1,2,1,-2,1} \\
& \left.+S_{2,1,1,-2,1}\right)+25 S_{2,-3,-2}+26\left(-S_{2,5}+S_{1,4,2}+S_{2,4,1}+S_{4,1,2}+S_{4,2,1}\right) \\
& +28\left(S_{1,2,4}+S_{2,1,4}-S_{-3,1,-2,1}-S_{-2,1,-3,1}-S_{1,-2,1,-3}\right)+30 S_{-3,1,-3} \\
& +32\left(S_{1,5,1}+S_{5,1,1}-S_{-3,-2,1,1}-S_{-2,-3,1,1}-S_{1,-3,-2,1}-S_{1,-2,-3,1}\right. \\
& \left.-S_{2,2,-2,1}+S_{1,2,-2,1,1}+S_{2,1,-2,1,1}-S_{1,1,1,-2,1,1}\right)+36\left(S_{1,1,5}+S_{1,3,-3}\right. \\
& +S_{3,1,-3}-S_{1,1,-3,-2}-S_{1,1,-2,-3}-S_{1,1,2,-3}-S_{1,2,-2,2}-S_{1,2,1,-3}-S_{2,1,-2,2} \\
& \left.-S_{2,1,1,-3}\right)+38 S_{-3,-3,1}+40\left(-S_{1,-4,1,1}-S_{2,-3,1,1}+S_{1,1,1,-2,2}\right) \\
& -41 S_{3,-4}+42\left(-S_{2,-5}+S_{1,-4,2}+S_{1,-3,-3}\right)+44\left(S_{1,-5,1}+S_{2,-3,2}+S_{3,-3,1}\right) \\
& +46 S_{2,2,-3}+48 S_{1,1,-3,1,1}+60\left(S_{1,1,-5}-S_{1,1,-3,2}\right)+62 S_{2,-4,1}+64 S_{1,1,1,-3,1} \\
& +68\left(S_{1,2,-4}+S_{2,1,-4}-S_{1,2,-3,1}-S_{2,1,-3,1}\right)-72 S_{1,1,1,-4}-80 S_{1,1,-4,1} \\
& -\zeta(3) S_{1}\left(S_{3}-S_{-3}+2 S_{-2,1}\right) \text {. }
\end{aligned}
$$

Table 1: The result for the four-loop asymptotic dimension $\frac{\gamma_{8}^{\mathrm{ABA}}(M)}{256}$. The harmonic sums are functions of $M$ and are defined in (2.5). The basis is canonical, except for the terms stemming from the dressing factor in the last line. 
the quantum corrections to the one-loop roots are of course linear, and thus numerically solvable with high precision.

Under the further assumption that no negative first index can appear in the harmonic sums (see [21] and discussion therein) there are, in principle, 238 terms which may potentially contribute to the four-loop dimension. One thus needs to solve the Bethe equations for 238 different values of spin $M$. In order to find the exact coefficients in front of the harmonic sums, which fortunately are integers, it is crucial to determine at each value of $M$ the anomalous dimension as a numerically exact rational number. One is thus forced to calculate with a very high numerical precision, i.e. one needs typically more than 1000 digits at four loop order. It is possible to reduce the number of the terms in the ansatz by going to a non-canonical basis of harmonic sums [25]. In the end one needs to determine around 170 values of $M$ from the Bethe equations. An important trick is to also use the information for odd values of $M$, even though these are unphysical. After much effort the expression given in table 1 was found. There we use the following definition of the harmonic sums [25]

$$
S_{a}(M)=\sum_{j=1}^{M} \frac{(\operatorname{sgn}(a))^{j}}{j^{a}}, \quad S_{a_{1}, \ldots, a_{n}}(M)=\sum_{j=1}^{M} \frac{\left(\operatorname{sgn}\left(a_{1}\right)\right)^{j}}{j^{a_{1}}} S_{a_{2}, \ldots, a_{n}}(j) .
$$

The degree of an harmonic sum is defined to be $\left|a_{1}\right|+\ldots\left|a_{n}\right|$. Notice that the total degree of each term in table 1 is seven in accordance with the maximal transcendentality principle. The expressions for the finite $M$ one-, two- and three-loop results are not reprinted here, they may be found in [20]. We have highlighted the terms in the last line, containing the number $\zeta(3)$ induced by the dressing factor (2.3). Using [26], we have also rewritten the result in an interesting non-canonical basis, see table 2 in appendix $\mathrm{A}$.

We should stress that our method, which might appear to be only approximately valid at first sight, actually leads to the exact perturbative solution of the Bethe equations. The reason is that a proper set of harmonic sums spans a linearly independent basis in a finite dimensional vector space [25]. A wrong ansatz produces incredibly complicated coefficients multiplying the harmonic sums, and breaks down immediately when compared with a further value of $M$ which was not yet matched.

We are now ready to analytically continue the expression in table 1 to the vicinity of the pomeron pole at $M=-1+\omega$. An explanation for how this is done may be found in [27]. It is based on a method suggested in [28], see also [29].

Harmonic sums of degree seven may lead to poles no higher than seventh order in $\omega$. In fact, it is known that none of the sums in table 1 can produce such a high-order pole except for the two sums $S_{7}$ and $S_{-7}$, which we have highlighted at the beginning of the table. Their residues at $1 / \omega^{7}$ are of opposite sign. Thus, one immediately sees that the sum of the two residues does not cancel. The precise statement was already quoted in (1.9). This proves our claim. 


\section{NLO BFKL and Double-Logarithm Constraints}

The asymptotic Bethe ansatz fails the BFKL constraint at four loops already to leading order in the expansion around the pomeron resonance singularity at $M=-1$ through the failure to cancel the erroneous seventh-order pole in $\omega, c f$ (1.9). The proper leading behavior (1.8) should definitely be quantitatively reproduced by any future proposal for exact spectral equations of AdS/CFT.

In fact, there are further known constraints from $\mathcal{N}=4$ high energy scattering amplitudes. Here we will state what is known, in order to provide precise tools for testing the validity of any future proposal for the exact spectrum. These highly non-trivial constraints fall into two classes: Next-to-leading order (NLO) corrections to the BFKL equation (1.6), and the so-called double-log predictions. Let us begin by discussing the former.

\subsection{Two-Loop BFKL}

We discussed in chapter 1 that the one-loop BFKL equation (1.6) leads to all-loop results for the leading singularities of the analytically continued anomalous dimensions of twisttwo operators at the special value $M=-1$. Likewise, the two-loop correction to the BFKL equation leads to constraints on the next-to-leading corrections to the position of the pomeron singularity near $M=-1$ [30]. Luckily this two-loop correction to the BFKL equation was worked out in the case of the $\mathcal{N}=4$ supersymmetric gauge theory [31, 21].

The two-loop corrected BFKL equation, $c f$ (1.6), for the twist-two case can be written in the dimensional reduction scheme as

$$
\frac{\omega}{-4 g^{2}}=\chi(\gamma)-g^{2} \delta(\gamma)
$$

where

$$
\begin{aligned}
\chi(\gamma)= & \Psi\left(-\frac{\gamma}{2}\right)+\Psi\left(1+\frac{\gamma}{2}\right)-2 \Psi(1) \\
\delta(\gamma)= & 4 \chi^{\prime \prime}(\gamma)+6 \zeta(3)+2 \zeta(2) \chi(\gamma)+4 \chi(\gamma) \chi^{\prime}(\gamma) \\
& -\frac{\pi^{3}}{\sin \frac{\pi \gamma}{2}}-4 \Phi\left(-\frac{\gamma}{2}\right)-4 \Phi\left(1+\frac{\gamma}{2}\right) .
\end{aligned}
$$

The function $\Phi(\gamma)$ is given by

$$
\Phi(\gamma)=\sum_{k=0}^{\infty} \frac{(-1)^{k}}{(k+\gamma)^{2}}[\Psi(k+\gamma+1)-\Psi(1)]
$$

This allows us, upon power series inversion, to compute the correction to the leading poles 
to arbitrary orders in $g$. In particular, the four-loop result (1.8) is extended to

$$
\begin{aligned}
\gamma=(2+0 \omega)\left(\frac{-4 g^{2}}{\omega}\right)-(0+0 \omega)\left(\frac{-4 g^{2}}{\omega}\right)^{2}+ & (0+\zeta(3) \omega)\left(\frac{-4 g^{2}}{\omega}\right)^{3} \\
& -\left(4 \zeta(3)+\frac{5}{4} \zeta(4) \omega\right)\left(\frac{-4 g^{2}}{\omega}\right)^{4} \pm \ldots
\end{aligned}
$$

\subsection{Double Logarithms}

The double-logarithmic asymptotics of the scattering amplitudes was investigated in QED and QCD in the papers [32] and [33] (see also [34]). It corresponds to summing the leading terms $\sim\left(\alpha \ln ^{2} s\right)^{n}$ in all orders of perturbation theory. In combination with a Mellin transformation, the double-logarithmic asymptotics allows to predict the singular part of anomalous dimensions near the point $M=-2$. According to the hypothesis formulated in the articles [31, 21], one can calculate the anomalous dimension $\gamma$ near other non-physical points $M=j-2=-r(r=2,3, \ldots)$ from the eigenvalue of the BFKL kernel

$$
\frac{\omega}{-4 g^{2}}=\Psi\left(-\frac{\gamma}{2}\right)+\Psi\left(1+\frac{\gamma}{2}+|n|\right)-2 \Psi(1)
$$

by pushing the total conformal spin $|n|$ to negative integer values $|n|=-r+1, r=2,3, \ldots$, rapidly enough at $\omega=M+r \rightarrow 0$ :

$$
|n|+r-1=C_{1}(r) \omega^{2}+\mathcal{O}\left(\omega^{3}\right) .
$$

Physically this corresponds to the double-logarithmic contributions $\sim\left(\alpha \ln ^{2} s\right)^{n} s^{-r+2}$ in the Regge limit $s \rightarrow \infty$. For even $r$ due to next-to-leading corrections the argument of the second $\Psi$-function in (3.6) is effectively shifted [31, 21]

$$
1+\frac{\gamma}{2}+|n| \rightarrow 1+\frac{\gamma}{2}+|n|+\omega
$$

and we derive the following equation for $\gamma$

$$
\gamma(2 \omega+\gamma)=-16 g^{2}
$$

The solution of this equation is

$$
\begin{aligned}
\gamma & =-\omega+\omega \sqrt{1-\frac{16 g^{2}}{\omega^{2}}} \\
& =2 \frac{\left(-4 g^{2}\right)}{\omega}-2 \frac{\left(-4 g^{2}\right)^{2}}{\omega^{3}}+4 \frac{\left(-4 g^{2}\right)^{3}}{\omega^{5}}-10 \frac{\left(-4 g^{2}\right)^{4}}{\omega^{7}}-\ldots
\end{aligned}
$$

Interestingly, our result in table 1 agrees at negative even integer values of the spin with the $-10 \frac{\left(-4 g^{2}\right)^{4}}{\omega^{7}}$ term of this expansion. This presumably means that the asymptotic expression 
is quite "close" to the true result, even though it clashes with the singularity at $M=-1$. We shall attempt to improve it by brute force in the next chapter.

For odd values of $M$ in accordance with [33] one can obtain the more complicated set of equations

$$
\begin{gathered}
\tilde{\gamma}(2 \omega+\tilde{\gamma})=-16 g^{2}-16 \frac{g^{2}}{\omega} \tilde{\gamma}_{8}, \\
\tilde{\gamma}_{8}\left(2 \omega+\tilde{\gamma}_{8}\right)=-8 g^{2}+4 g^{2} \frac{d}{d \omega} \tilde{\gamma}_{8},
\end{gathered}
$$

where $\tilde{\gamma}_{8}$ is the anomalous dimension for an auxiliary operator with octet color quantum numbers. The solution of these equations coincides with the Born result

$$
\tilde{\gamma}_{8}=-4 \frac{g^{2}}{\omega}, \tilde{\gamma}=-8 \frac{g^{2}}{\omega}
$$

corresponding to the fact that at $r=2 k-1$ the leading terms $\sim \frac{g^{2}}{\omega}(g / \omega)^{2 n}$ are absent.

One can generalize the double-logarithmic equation (3.9) for even $r=2,4, \ldots$ to include the corrections reproducing the three leading poles up to third order in perturbation theory

$$
\gamma(2 \omega+\gamma)=-16 g^{2}\left(1-S_{1} \omega-\left(S_{2}+\zeta_{2}\right) \omega^{2}\right)-64 g^{4}\left(S_{2}+\zeta_{2}-S_{1}^{2}\right)+\left(S_{2}+S_{-2}\right) \mathcal{O}\left(g^{6}\right),
$$

where $S_{i}=S_{i}(r-1)$. The third order term $\mathcal{O}\left(g^{6}\right)$ has the form

$$
\mathcal{O}\left(g^{6}\right)=a_{1} g^{2} \gamma^{2}+a_{2} g^{4} \gamma_{8} / \omega
$$

where $a_{1,2}$ are some parameters. Choosing these parameters in an appropriate way, we can obtain from the solution of the above equation the correct residues of $\gamma$ for the strongest poles at even negative $M$ up to three loops. This predicts the corresponding residues to fourth order

$$
\gamma=2 \sum_{r=1}^{\infty} c_{r}(\omega)\left(-4 g^{2}\right)^{r}
$$

where

$$
\begin{aligned}
& c_{1}(\omega)=\frac{1}{\omega}-S_{1}-\omega\left(\zeta_{2}+S_{2}\right)+\ldots, \\
& c_{2}(\omega)=-\frac{1}{\omega^{3}}+\frac{2 S_{1}}{\omega^{2}}+\frac{\zeta_{2}+S_{2}}{\omega}+\ldots, \\
& c_{3}(\omega)=\frac{2}{\omega^{5}}-\frac{6 S_{1}}{\omega^{4}}+\frac{-4\left(\zeta_{2}+S_{2}\right)+4 S_{1}^{2}+\left(S_{2}+S_{-2}\right)}{\omega^{3}}+\ldots, \\
& c_{4}(\omega)=-\frac{5}{\omega^{7}}+\frac{20 S_{1}}{\omega^{6}}+\frac{14\left(\zeta_{2}+S_{2}\right)-24 S_{1}^{2}-4\left(S_{2}+S_{-2}\right)}{\omega^{5}}+\ldots
\end{aligned}
$$

Note, that for odd negative values of $M$ the generalized set of equations (3.11) containing the next-to-leading corrections has more parameters. One can fix some of them from the known singularities of the anomalous dimensions at $M+r=\omega \rightarrow 0(r=1,3, \ldots)$

$$
\gamma=-8 g^{2}\left(\frac{1}{\omega}-S_{1}-\omega\left(\zeta_{2}+S_{2}\right)\right)-32 g^{4} \frac{S_{2}}{\omega}-128 g^{6}\left(\frac{2 S_{1}}{\omega^{4}}+\frac{2 S_{2}+S_{-2}-2 S_{1}^{2}}{\omega^{3}}\right),
$$

but it is difficult to predict the residues of the corresponding poles in the fourth order. 


\section{Ad Hoc Improvement of Four-Loop Twist-Two}

Here we attempt to experimentally improve the erroneous four-loop result of table 1 as obtained by the dressed asymptotic Bethe ansatz such that all BFKL and double-logarithm constraints of section 3 are satisfied. Obviously this has to be done in a way which does not ruin the correct features of the expression in table 1. In particular, the improvement should not modify the large spin limit nor violate the transcendentality principle. A seemingly natural way to ensure this is to replace the explicit $\zeta(3)$ stemming from the dressing factor by an appropriate linear combination of $\zeta(3)$ and finite harmonic sums of degree three. We found that there is indeed an attractive choice, namely replacing in the last line of the expression in table $1 \zeta(3)$ by

$$
\zeta(3) \rightarrow \frac{47}{24} \zeta(3)-\frac{1}{4} S_{-3}+\frac{3}{4} S_{-2} S_{1}+\frac{3}{8} S_{1} S_{2}+\frac{3}{8} S_{3}+\frac{1}{6} S_{-2,1}-\frac{17}{24} S_{2,1} .
$$

This alteration clearly preserves transcendentality, and it is easy to check that the large spin limit is not modified. In addition, the catastrophic behavior in (1.9) is now replaced by the correct one in (1.8). Furthermore, the constraints from (3.5) and (3.16) are also satisfied 2 . In principle there are however other possible modifications which also fit all known data. We therefore stop short of calling the expression of table 1 with the replacement (4.1) a "conjecture".

It is nevertheless interesting to work out the four-loop anomalous dimension of the operator of lowest twist $M=2$, i.e. the Konishi field, by using the formula in table 1 with the replacement (4.1). One finds

$$
\gamma=12 g^{2}-48 g^{4}+336 g^{6}-\left(\frac{5307}{2}+564 \zeta(3)\right) g^{8}+\ldots
$$

These numbers should be compared to the ones at the end of chapter 5 of [15]. Once again, (4.2) is a result based on some reasonable but presumably not unique assumptions and we do not dare calling it a conjecture.

\section{Four-Loop Twist-Three from Bethe Ansatz}

In this article we are mostly focusing on twist-two operators. These are ideally suited for an analysis of the wrapping problem. Furthermore, their precise relationship to the BFKL equation is well established. Lastly, their spectrum may be exactly found at one-loop order. This yields a firm platform for higher orders of perturbation theory. However, all this does of course not mean that operators of higher twist are not interesting. Here we will report on a novel exact twist-three one-loop solution in the $\mathcal{N}=4$ model. This allows to find exact higher-loop anomalous dimensions in terms of nested harmonic sums, in close analogy with the twist-two case. Unfortunately we were so far unable to test the analytic continuation

\footnotetext{
${ }^{2}$ In the case of negative odd $M$ the situation is unclear.
} 
of these expressions with the BFKL and double-logarithm methods of chapter 3. We still feel that our result should allow for some non-trivial tests in the future.

Twist-three operators are operators of the form

$$
\operatorname{Tr}\left(D^{s_{1}} \mathcal{Z} \mathcal{D}^{s_{2}} \mathcal{Z} \mathcal{D}^{s_{3}} \mathcal{Z}\right)+\ldots
$$

with $s_{1}+s_{2}+s_{3}=M$. Since the wrapping effects in $\mathfrak{s l}(2)$ start at $\mathcal{O}\left(g^{2 L+4}\right)$ the four-loop anomalous dimension of twist-three operators should correctly follow from the asymptotic Bethe ansatz (2.1) with $J=3$. In this chapter we will proceed with the derivation of the four-loop anomalous dimension of the ground state of twist-three operators at even values of $M$. It will be shown that the anomalous dimension up to four-loop order can be again given in terms of harmonic sums, similarly to the twist-two case. After analytical continuation it will turn out, however, that the anomalous dimension does not have a pole at $M=-1$ and thus cannot be checked with the BFKL equation. The validity of this result as derived from the Bethe ansatz is therefore still an open question.

At one-loop the Baxter function for the twist-three operators satisfies

$$
\left(u+\frac{i}{2}\right)^{3} Q(u+i)+\left(u-\frac{i}{2}\right)^{3} Q(u-i)=t(u) Q(u),
$$

with the transfer matrix given by

$$
t(u)=2 u^{3}+q_{2} u+q_{3} .
$$

Using the expansion

$$
Q(u)=\prod_{n=1}^{M}\left(u-u_{i}\right)=u^{M}+c_{1} u^{M-1}+c_{2} u^{M-2}+c_{3} u^{M-3}+\ldots
$$

one can read off the corresponding charges

$$
q_{2}=-\left(M^{2}+2 M+\frac{3}{2}\right), \quad q_{3}=c_{1}(2 M+1) .
$$

For the unpaired states $c_{1}=-\sum_{j=1}^{M} u_{j}=0$ and $q_{3}=0$. One can then solve (5.2) exactly for even values of $M$

$$
Q(u)={ }_{4} F_{3}\left(-\frac{M}{2}, \frac{M}{2}+1, \frac{1}{2}+i u, \frac{1}{2}-i u, ; 1,1,1 ; 1\right) .
$$

Thus the Baxter function $Q(u)$ is given by a Wilson polynomial. Wilson polynomials for twist-three operators were also found in the QCD context, see [35]. It is straightforward to derive the corresponding one-loop anomalous dimension

$$
\frac{\gamma_{2}^{\mathrm{ABA}}(M)}{2}=4 S_{1}\left(\frac{M}{2}\right)
$$


There exists one more solution of (5.2) for $q_{0}=0$ which is, however, non-polynomial. This proves that all unpaired states for even $M$ have energy given by (5.7). We suspect that this is the lowest state, but we do not know any proof.

Similarly to the twist-two case one can derive from the Bethe ansatz a closed formula for the corresponding two, three and four-loop anomalous dimensions by assuming the transcendentality principle and making an appropriate ansatz. We found the following expressions, where all harmonic sums have $\frac{M}{2}$ as an argument

$$
\begin{aligned}
& \frac{\gamma_{4}^{\mathrm{ABA}}(M)}{4}=-2 S_{3}-4 S_{1} S_{2} \\
& \frac{\gamma_{6}^{\mathrm{ABA}}(M)}{8}=2 S_{2} S_{3}+S_{5}+4 S_{3,2}+4 S_{4,1}-8 S_{3,1,1}+S_{1}\left(4 S_{2}^{2}+2 S_{4}+8 S_{3,1}\right) \\
& \frac{\gamma_{8}^{\mathrm{ABA}}(M)}{16}= S_{1}^{3}\left(\frac{40}{3} S_{4}-\frac{32}{3} S_{3,1}\right)+S_{1}^{2}\left(20 S_{5}-40 S_{3,2}-56 S_{4,1}+64 S_{3,1,1}\right) \\
&+ S_{1}\left(7 S_{6}+8 S_{2,4}-24 S_{3,3}-56 S_{4,2}-40 S_{5,1}-24 S_{2,2,2}-16 S_{2,3,1}\right. \\
&\left.+88 S_{3,1,2}+88 S_{3,2,1}+120 S_{4,1,1}-192 S_{3,1,1,1}-8 \zeta(3) S_{3}\right)-\frac{56}{3} S_{3} S_{4} \\
&-\frac{107}{6} S_{7}+3 S_{2,5}+\frac{41}{3} S_{3,4}+\frac{1}{3} S_{4,3}-17 S_{5,2}-\frac{20}{3} S_{6,1}-4 S_{2,2,3} \\
&-8 S_{2,3,2}-4 S_{2,4,1}+\frac{104}{3} S_{3,1,3}+52 S_{3,2,2}+\frac{88}{3} S_{3,3,1}+60 S_{4,1,2} \\
&+60 S_{4,2,1}+40 S_{5,1,1}+8 S_{2,3,1,1}-120 S_{3,1,1,2}-120 S_{3,1,2,1} \\
&-120 S_{3,2,1,1}-128 S_{4,1,1,1}+256 S_{3,1,1,1,1} .
\end{aligned}
$$

It is also instructive to display $\gamma_{8}$ in the canonical basis of harmonic sums

$$
\begin{aligned}
\frac{\gamma_{8}^{\mathrm{ABA}}(M)}{16}= & \frac{1}{2} S_{7}+7 S_{1,6}-5 S_{6,1}-5 S_{3,4}-29 S_{4,3}-32 S_{1,2,4}-32 S_{2,1,4}+32 S_{1,4,2} \\
& +4 S_{2,4,1}+36 S_{4,1,2}+36 S_{4,2,1}-24 S_{1,4,1,1}-24 S_{4,1,1,1}+15 S_{2,5}-21 S_{5,2} \\
& +24 S_{5,1,1}+24 S_{3,3,1}+24 S_{3,1,3}+24 S_{1,3,3}+44 S_{3,2,2}+40 S_{2,3,2}+20 S_{2,2,3} \\
& -24 S_{1,3,1,2}+16 S_{1,2,3,1}-24 S_{1,3,2,1}-24 S_{3,1,1,2}+16 S_{2,1,3,1}-24 S_{3,1,2,1} \\
& -24 S_{2,3,1,1}-24 S_{3,2,1,1}-24 S_{1,2,2,2}-24 S_{2,1,2,2}-24 S_{2,2,1,2}-24 S_{2,2,2,1} \\
& -40 S_{1,1,5}+80 S_{1,1,1,4}+32 S_{1,1,4,1}-16 S_{1,1,3,2}-64 S_{1,1,1,3,1} \\
& -\mathbf{8} \boldsymbol{\zeta}(\mathbf{3})\left(\boldsymbol{S}_{\mathbf{1 , 3}}+\boldsymbol{S}_{\mathbf{3 , 1}}-\boldsymbol{S}_{\mathbf{4}}\right) .
\end{aligned}
$$

The term multiplied by $\zeta(3)$ is due to the dressing factor. Curiously, only positive indices in the harmonic sums appear. Because the argument of the harmonics sums in (5.7)(5.10) is $\frac{M}{2}$, there is no pole at $M=-1$ and thus these states are not captured by the BFKL equation. It would be very interesting to see whether one can predict their analytic 
structure at $M \leq-2$ from the double-log constraints. We would like to stress again the fact that equations (5.7)-(5.10), even when the asymptotic character of the Bethe equations (2.1) is taken into account, should give correct anomalous dimensions. Below we present the two highest terms in the analytical continuation to $M=2(\omega-1)$

$$
\begin{array}{lll}
\frac{\gamma_{2}^{\mathrm{ABA}}}{2}=-\frac{4}{\omega}+4 \zeta(2) \omega+\ldots, & \frac{\gamma_{4}^{\mathrm{ABA}}}{4}=-\frac{2}{\omega^{3}}+\frac{4}{\omega} \zeta(2)+\ldots, \\
\frac{\gamma_{6}^{\mathrm{ABA}}}{8}=-\frac{1}{\omega^{5}}+\frac{6}{\omega^{3}} \zeta(2)+\ldots, & \frac{\gamma_{8}^{\mathrm{ABA}}}{16}=-\frac{1}{2 \omega^{7}}+\frac{5}{\omega^{5}} \zeta(2) \ldots .
\end{array}
$$

Note that the leading singularity is the same for all points $M=2(\omega-(k+1))$ and $k=0,1, \ldots$. The corresponding expansion of the total anomalous dimension up to fourloop order reads

$$
\begin{aligned}
\gamma^{\mathrm{ABA}}= & -8 \frac{g^{2}}{\omega}\left[1-\zeta(2) \omega^{2}+t\left(1-2 \zeta(2) \omega^{2}\right)+t^{2}\left(1-6 \zeta(2) \omega^{2}\right)\right. \\
& \left.+t^{3}\left(1-10 \zeta(2) \omega^{2}\right)+\ldots\right]+\ldots
\end{aligned}
$$

where

$$
t=\frac{g^{2}}{\omega^{2}}
$$

One can speculate on an all-loop generalization of (5.13). A plausible form might be

$$
\gamma^{\mathrm{ABA}}=-8 \frac{g^{2}}{\omega}\left(\frac{1}{1-t}-\zeta(2) \frac{1+3 t^{2}}{(1-t)^{2}} \omega^{2}+\ldots\right)+\ldots
$$

It is interesting to note that the double-logarithmic behavior of these states is different from the twist-two ones (3.10).

\section{Outlook}

Our result in table 1 with the ensuing (1.9) proves unequivocally that the spectral equations of [9, 15] for AdS/CFT are still incomplete as the BFKL prediction (1.8) is not reproduced at four-loop order $\mathcal{O}\left(g^{8}\right)$.

Our weak-coupling study is complementary to indications that at strong coupling, i.e. on the string side of the AdS/CFT correspondence, the asymptotic Bethe ansatz [9, 15] is also incomplete when one considers finite size effect of the string worldsheet. For one, it was argued that for a specific spinning string solution carrying both angular momentum w.r.t. $A d S_{5}$ and $S^{5}$ as well as winding numbers, and whose classical [36] and one-loop [37] energy is known, the ansatz does not reproduce exponentially small terms in the size of the system [38]. A second indication comes from a study of the finite size effects [39] on the dispersion law of classical giant magnons [40]. Again, terms which fall off exponentially 
with the volume are seen which cannot easily be accounted for by the dressed asymptotic spectral equations.

We have shown that linking the integrable structures found in the context of high energy scattering amplitudes in $\mathcal{N}=4$ theory and the ones appearing in the spectral problem leads to very strong constraints. It was pointed out in [20] and [41] that pomeron physics and anomalous dimensions are very naturally connected through the AdS/CFT correspondence. However, no attention had so far been payed to the fact that integrability will presumably allow to truly explore these connections in a quantitative and analytic fashion. We feel that we have made a first step in this direction.

Interestingly, the breakdown we observe is completely insensitive to the structure of the dressing factor, which also appears at four-loop order [15], in contradistinction to what one might have hoped for. Recall that this dressing phase leads to the four-loop agreement between the Bethe ansatz and the result of a gluon scattering amplitude in $\mathcal{N}=4$ theory [13, 16, 15]. This is not contradictory. The gluon amplitude tests the anomalous dimension in the large spin limit $M \rightarrow \infty$, where it was argued that the all-loop result leads to a universal scaling function [14] (see also [42]), i.e. one which is reached by large $M$ scaling of the lowest state at fixed length=twist. We may therefore choose the twist large enough to avoid leaving the asymptotic regime. Turning this around, we might say that the wrapping terms should be subleading in the large spin limit. This is reassuring, in particular since by now it appears that the scaling function matches well [43] the known string theory results [44]. Furthermore, the analytic structure of the dressing phase fits well a semi-classical analysis [45].

In this article it is proven that wrapping effects are not properly taken into account by the existing asymptotic Bethe ansatz. We note that this was of course never claimed otherwise by any of the current authors, and is actually quite expected from the way this Bethe ansatz was initially constructed [7]. The mechanism for the breakdown of the asymptotic approximation should be similar to the one discussed from a field theory standpoint in [46], and from a lattice model point of view in [47].

\section{Acknowledgments}

A.R. and M.S. would like to thank Lisa Freyhult, Romuald Janik and Stefan Zieme for useful discussions. L.N.L. thanks Yu. Dokshitzer for helpful discussions of the analytic properties of the anomalous dimensions of Wilson operators with twists two and three. L.N.L. holds a Marie Curie Chair of Excellence and acknowledges the support of the European Commission. A.V.K. is supported in part by the Alexander von Humboldt Foundation. L.N.L. is supported by 06-02-72041-MSTI-a, V.N.V. is supported by DFG grant No. KN 365/6-1, and A.V.K., L.N.L. and V.N.V. are supported by RFBR grants

07-02-00902-a, RSGSS-5788.2006.2. M.S. and A.R. thank the Jagiellonian University in Kraków for hospitality while working on parts of this project. 


\section{A Asymptotic Four-Loop Anomalous Dimension of Twist-Two Operators in a Non-Canonical Basis}

$$
\begin{aligned}
& -8 S_{-3}\left(4 S_{-4}+S_{-2}^{2}+2 S_{-2} S_{2}+2 S_{2}^{2}+3 S_{4}+2 S_{-2,2}+8 S_{-2,1,1}\right) \\
& -16 S_{-2,1}\left(4 S_{-4}+S_{-2}^{2}-2 S_{-2} S_{2}-2 S_{2}^{2}-5 S_{4}+6 S_{-2,2}+8 S_{-2,1,1}\right) \\
& -\frac{8}{3} S_{3}\left(36 S_{-4}+9 S_{-2}^{2}+18 S_{-2} S_{2}+6 S_{2}^{2}+9 S_{4}-56 S_{-3,1}-50 S_{-2,2}\right. \\
& \left.+88 S_{-2,1,1}\right)-16 S_{2}\left(3 S_{-5}+S_{5}-6\left(S_{-4,1}+S_{-3,2}+S_{-2,3}-2 S_{-3,1,1}\right.\right. \\
& \left.\left.-2 S_{-2,1,2}-2 S_{-2,2,1}+4 S_{-2,1,1,1}\right)\right)-32 S_{-2}\left(4 S_{-5}+2 S_{5}-S_{-4,1}\right. \\
& \left.-S_{-2,3}+S_{4,1}-2 S_{-2,-2,1}\right)-\frac{32}{3} S_{1}^{3}\left(18 S_{-4}-3 S_{-2}^{2}+3 S_{4}-16 S_{-3,1}\right. \\
& \left.-10 S_{-2,2}+8 S_{-2,1,1}\right)-32 S_{1}^{2}\left(S_{-2}\left(5 S_{-3}+3 S_{3}+2 S_{-2,1}\right)\right. \\
& +3 S_{2}\left(3 S_{-3}+S_{3}-2 S_{-2,1}\right)+15 S_{-5}+5 S_{5}-2\left(10 S_{-4,1}+S_{-3,-2}\right. \\
& +10 S_{-3,2}+7 S_{-2,3}-S_{4,1}-14 S_{-3,1,1}+2 S_{-2,-2,1}-10 S_{-2,1,2}-10 S_{-2,2,1} \\
& \left.\left.+12 S_{-2,1,1,1}\right)\right)-16 S_{1}\left(S_{3}\left(12 S_{-3}-12 S_{-2,1}\right)+S_{-2}\left(8 S_{-4}+6 S_{2}^{2}+9 S_{4}\right.\right. \\
& \left.-12 S_{-3,1}-2 S_{-2,2}\right)+2 S_{2}\left(8 S_{-4}+2 S_{-2}^{2}+3 S_{4}-12 S_{-3,1}-10 S_{-2,2}\right. \\
& \left.+16 S_{-2,1,1}\right)+26 S_{-6}-3 S_{-3}^{2}+S_{-2}^{3}+2 S_{2}^{3}+2 S_{3}^{2}+3 S_{6}-44 S_{-5,1} \\
& -46 S_{-4,2}-46 S_{-3,3}-4 S_{-2,1}^{2}-38 S_{-2,4}+4 S_{4,2}-8 S_{5,1}+80 S_{-4,1,1} \\
& +8 S_{-3,-2,1}+8 S_{-3,1,-2}+84 S_{-3,1,2}+84 S_{-3,2,1}-8 S_{-2,-2,2}+68 S_{-2,1,3} \\
& +72 S_{-2,2,2}+68 S_{-2,3,1}-8 S_{4,1,1}-144 S_{-3,1,1,1}+16 S_{-2,-2,1,1}-120 S_{-2,1,1,2} \\
& \left.-120 S_{-2,1,2,1}-120 S_{-2,2,1,1}+192 S_{-2,1,1,1,1}\right)-16\left(8 S_{-7}+9 S_{7}-16 S_{-6,1}\right. \\
& -6 S_{-5,-2}-16 S_{-5,2}-S_{-4,-3}-17 S_{-4,3}-15 S_{-3,4}-18 S_{-2,5}-5 S_{4,3} \\
& +4 S_{5,2}+6 S_{6,1}+32 S_{-5,1,1}-6 S_{-4,-2,1}+36 S_{-4,1,2}+36 S_{-4,2,1} \\
& -4 S_{-3,-3,1}-2 S_{-3,-2,-2}-4 S_{-3,-2,2}+36 S_{-3,1,3}+40 S_{-3,2,2}+36 S_{-3,3,1} \\
& +2 S_{-2,-4,1}-8 S_{-2,-3,2}+10 S_{-2,-2,3}+34 S_{-2,1,4}+36 S_{-2,2,3}+36 S_{-2,3,2} \\
& +32 S_{-2,4,1}-4 S_{4,1,2}-4 S_{4,2,1}-72 S_{-4,1,1,1}-80 S_{-3,1,1,2}-80 S_{-3,1,2,1} \\
& -80 S_{-3,2,1,1}+24 S_{-2,-3,1,1}+4 S_{-2,-2,-2,1}+8 S_{-2,-2,1,2}+8 S_{-2,-2,2,1} \\
& -8 S_{-2,1,1,-3}-72 S_{-2,1,1,3}-80 S_{-2,1,2,2}-72 S_{-2,1,3,1}-8 S_{-2,2,-2,1} \\
& -80 S_{-2,2,1,2}-80 S_{-2,2,2,1}-72 S_{-2,3,1,1}+24 S_{4,1,1,1}+160 S_{-3,1,1,1,1} \\
& -48 S_{-2,-2,1,1,1}-16 S_{-2,1,-2,1,1}+160 S_{-2,1,1,1,2}+160 S_{-2,1,1,2,1} \\
& \left.+160 S_{-2,1,2,1,1}+160 S_{-2,2,1,1,1}-320 S_{-2,1,1,1,1,1}\right) \\
& -16 \zeta(3) S_{1}\left(S_{3}-S_{-3}+2 S_{-2,1}\right)
\end{aligned}
$$

Table 2: The four-loop asymptotic dimension $\frac{\gamma_{8}^{\mathrm{ABA}}(M)}{16}$ of table 1 in a non-canonical basis. This isolates the $S_{1}$, i.e all terms divergent as $M \rightarrow \infty$. 


\section{References}

[1] L. N. Lipatov, "High-energy asymptotics of multicolor QCD and exactly solvable lattice models," (unpublished), arXiv:hep-th/9311037.

[2] L. N. Lipatov, "Asymptotic behavior of multicolor QCD at high energies in connection with exactly solvable spin models," JETP Lett. 59 (1994) 596 [Pisma Zh. Eksp. Teor. Fiz. 59 (1994) 571]. • L. D. Faddeev and G. P. Korchemsky, "High-energy QCD as a completely integrable model," Phys. Lett. B 342 (1995) 311, arXiv:hep-th/9404173.

[3] L. N. Lipatov, "Evolution equations in QCD", in "Perspectives in Hadronic Physics," Proceedings of the Conference, ICTP, Trieste, Italy, 12-16 May 1997, eds. S. Boffi, C. Ciofi Degli Atti and M. Giannini, World Scientific (Singapore, 1998).

[4] V. M. Braun, S. E. Derkachov and A. N. Manashov, "Integrability of three-particle evolution equations in QCD," Phys. Rev. Lett. 81 (1998) 2020, arXiv: hep-ph/9805225. • V. M. Braun, S. E. Derkachov, G. P. Korchemsky and A. N. Manashov, "Baryon distribution amplitudes in QCD," Nucl. Phys. B 553 (1999) 355, arXiv:hep-ph/9902375. - A. V. Belitsky, "Fine structure of spectrum of twist-three operators in QCD," Phys. Lett. B 453 (1999) 59, arXiv:hep-ph/9902361. • A. V. Belitsky, "Renormalization of twist-three operators and integrable lattice models," Nucl. Phys. B 574 (2000) 407, arXiv: hep-ph/9907420.

[5] J. A. Minahan and K. Zarembo, "The Bethe ansatz for $\mathcal{N}=4$ super Yang-Mills", JHEP 0303 (2003) 013, arXiv:hep-th/0212208. • N. Beisert and M. Staudacher, "The $\mathcal{N}=4$ SYM integrable super spin chain", Nucl. Phys. B 670, 439 (2003), arXiv:hep-th/0307042.

[6] N. Beisert, C. Kristjansen and M. Staudacher, "The dilatation operator of $\mathcal{N}=4$ super Yang-Mills theory," Nucl. Phys. B 664 (2003) 131, arXiv:hep-th/0303060.

N. Beisert, "The $\mathfrak{s u}(2 \mid 3)$ dynamic spin chain," Nucl. Phys. B 682 (2004) 487, arXiv: hep-th/0310252. • D. Serban and M. Staudacher, "Planar $\mathcal{N}=4$ gauge theory and the Inozemtsev long range spin chain", JHEP 0406, 001 (2004),

arXiv:hep-th/0401057. • N. Beisert, V. Dippel and M. Staudacher, "A novel long range spin chain and planar $\mathcal{N}=4$ super Yang-Mills", JHEP 0407, 075 (2004), arXiv:hep-th/0405001.

[7] M. Staudacher, "The factorized S-matrix of CFT/AdS", JHEP 0505, 054 (2005), arXiv:hep-th/0412188.

[8] N. Beisert, "The $\mathfrak{s u}(2 \mid 2)$ dynamic S-matrix," arXiv:hep-th/0511082.

[9] N. Beisert and M. Staudacher, "Long-range $\mathfrak{p s u}(2,2 \mid 4)$ Bethe ansätze for gauge theory and strings", Nucl. Phys. B 727 (2005) 1, arXiv:hep-th/0504190. 
[10] I. Bena, J. Polchinski and R. Roiban, "Hidden symmetries of the $A d S_{5} \times S^{5}$ superstring," Phys. Rev. D 69 (2004) 046002, arXiv:hep-th/0305116. • V. A. Kazakov, A. Marshakov, J. A. Minahan and K. Zarembo, "Classical / quantum integrability in AdS/CFT, ” JHEP 0405 (2004) 024, arXiv: hep-th/0402207.

[11] G. Arutyunov, S. Frolov and M. Staudacher, "Bethe ansatz for quantum strings," JHEP 0410 (2004) 016, arXiv:hep-th/0406256.

[12] R. A. Janik, "The AdS $S_{5} \times S^{5}$ superstring worldsheet $S$-matrix and crossing symmetry", Phys. Rev. D 73 (2006) 086006, arXiv:hep-th/0603038. • R. Hernandez and E. Lopez, "Quantum corrections to the string Bethe ansatz," JHEP 0607 (2006) 004, arXiv:hep-th/0603204. • G. Arutyunov and S. Frolov, "On $A d S_{5} x \times S^{5}$ string $S$ matrix," Phys. Lett. B 639 (2006) 378, arXiv:hep-th/0604043. • N. Beisert, R. Hernandez and E. Lopez, "A crossing-symmetric phase for $A d S_{5} \times S^{5}$ strings," JHEP 0611 (2006) 070, arXiv:hep-th/0609044.

[13] Z. Bern, M. Czakon, L. J. Dixon, D. A. Kosower and V. A. Smirnov, "The fourloop planar amplitude and cusp anomalous dimension in maximally supersymmetric Yang-Mills theory", arXiv:hep-th/0610248.

[14] B. Eden and M. Staudacher, "Integrability and transcendentality," J. Stat. Mech. 0611 (2006) P014, arXiv:hep-th/0603157.

[15] N. Beisert, B. Eden and M. Staudacher, "Transcendentality and crossing," J. Stat. Mech. 0701 (2007) P021, arXiv:hep-th/0610251.

[16] F. Cachazo, M. Spradlin and A. Volovich, "Four-loop cusp anomalous dimension from obstructions", arXiv:hep-th/0612309.

[17] A. Rej, M. Staudacher and S. Zieme, "Nesting and dressing," arXiv:hep-th/0702151.

[18] N. Gromov and P. Vieira, "Constructing the AdS/CFT dressing factor," arXiv:hep-th/0703266.

[19] A. V. Kotikov, L. N. Lipatov and V. N. Velizhanin, "Anomalous dimensions of Wilson operators in $N=4$ SYM theory," Phys. Lett. B 557 (2003) 114, arXiv:hep-ph/0301021.

[20] A. V. Kotikov, L. N. Lipatov, A. I. Onishchenko and V. N. Velizhanin, "Threeloop universal anomalous dimension of the Wilson operators in $N=4 S U S Y$ YangMills model," Phys. Lett. B 595 (2004) 521 [Erratum-ibid. B 632 (2006) 754], arXiv: hep-th/0404092.

[21] A. V. Kotikov and L. N. Lipatov, "DGLAP and BFKL equations in the $N=4$ supersymmetric gauge theory," Nucl. Phys. B 661 (2003) 19 [Erratum-ibid. B 685 (2004) 405], arXiv:hep-ph/0208220. 
[22] S. Moch, J. A. M. Vermaseren and A. Vogt, "The three-loop splitting functions in QCD: The non-singlet case," Nucl. Phys. B 688 (2004) 101, arXiv:hep-ph/0403192.

[23] Yu. L. Dokshitzer, G. Marchesini and G. P. Salam, "Revisiting parton evolution and the large-x limit," Phys. Lett. B 634 (2006) 504, arXiv:hep-ph/0511302. • Yu. L. Dokshitzer and G. Marchesini, "N = 4 SUSY Yang-Mills: Three loops made simple(r)," Phys. Lett. B 646 (2007) 189, arXiv:hep-th/0612248. • B. Basso and G. P. Korchemsky, "Anomalous dimensions of high-spin operators beyond the leading order," arXiv: hep-th/0612247.

[24] L. N. Lipatov, "Reggeization of the vector meson and the vacuum singularity in nonabelian gauge theories," Sov. J. Nucl. Phys. 23 (1976) 338 [Yad. Fiz. 23 (1976) 642]. - E. A. Kuraev, L. N. Lipatov and V. S. Fadin, "The Pomeranchuk singularity in nonabelian gauge theories," Sov. Phys. JETP 45 (1977) 199 [Zh. Eksp. Teor. Fiz. 72 (1977) 377]. • I. I. Balitsky and L. N. Lipatov, "The Pomeranchuk singularity in Quantum Chromodynamics,” Sov. J. Nucl. Phys. 28 (1978) 822 [Yad. Fiz. 28 (1978) 1597].

[25] J. A. M. Vermaseren, "Harmonic sums, Mellin transforms and integrals," Int. J. Mod. Phys. A 14 (1999) 2037, arXiv:hep-ph/9806280.

[26] J. Blümlein, "Algebraic relations between harmonic sums and associated quantities," Comput. Phys. Commun. 159 (2004) 19, arXiv: hep-ph/0311046.

[27] A. V. Kotikov and V. N. Velizhanin, "Analytic continuation of the Mellin moments of deep inelastic structure functions," arXiv:hep-ph/0501274.

[28] A. Gonzalez-Arroyo, C. Lopez and F. J. Yndurain, "Second order contributions to the structure functions in deep inelastic scattering. 1. Theoretical calculations," Nucl. Phys. B 153 (1979) 161.

[29] D. I. Kazakov and A. V. Kotikov, "Total alpha-s correction to deep inelastic scattering cross-section ratio, $R=\sigma_{L} / \sigma_{t}$ in $Q C D$. Calculation of longitudinal structure function," Nucl. Phys. B 307 (1988) 721 [Erratum-ibid. B 345 (1990) 299]. • A. V. Kotikov, "Gluon distribution for small x," Phys. Atom. Nucl. 57 (1994) 133 [Yad. Fiz. 57 (1994) $142]$.

[30] V. S. Fadin and L. N. Lipatov, "BFKL pomeron in the next-to-leading approximation," Phys. Lett. B 429 (1998) 127, arXiv: hep-ph/9802290. • M. Ciafaloni and G. Camici, "Energy scale(s) and next-to-leading BFKL equation," Phys. Lett. B 430 (1998) 349, arXiv: hep-ph/9803389.

[31] A. V. Kotikov and L. N. Lipatov, "NLO corrections to the BFKL equation in $Q C D$ and in supersymmetric gauge theories,"

Nucl. Phys. B 582 (2000) 19, arXiv:hep-ph/0004008. 
[32] V. G. Gorshkov, V. N. Gribov, L. N. Lipatov and G. V. Frolov, "Doubly logarithmic asymptotic behavior in Quantum Electrodynamics," Sov. J. Nucl. Phys. 6 (1968) 95 [Yad. Fiz. 6 (1967) 129]; "Backward Electron - Positron scattering at high energies," Sov. J. Nucl. Phys. 6 (1968) 262 [Yad. Fiz. 6 (1967) 361].

[33] R. Kirschner and L. N. Lipatov, "Doubly logarithmic asymptotic of the quark scattering amplitude with nonvacuum exchange in the t-channel," Sov. Phys. JETP 56 (1982) 266 [Zh. Eksp. Teor. Fiz. 83 (1982) 488]; "Double logarithmic asymptotics of quark scattering amplitudes with flavor exchange," Phys. Rev. D 26 (1982) 1202; "Double logarithmic asymptotics and Regge singularities of quark amplitudes with flavor exchange," Nucl. Phys. B 213 (1983) 122.

[34] J. Bartels, B. I. Ermolaev and M. G. Ryskin, "Nonsinglet contributions to the structure function $g_{1}$ at small $x$," Z. Phys. C 70, 273 (1996), arXiv:hep-ph/9507271; "Flavor singlet contribution to the structure function $g_{1}$ at small $x$," Z. Phys. C 72, 627 (1996) arXiv:hep-ph/9603204.

[35] G. P. Korchemsky, "Bethe Ansatz for QCD Pomeron," Nucl. Phys. B 443 (1995) 255, arXiv:hep-ph/9501232.

[36] G. Arutyunov, J. Russo and A. A. Tseytlin, "Spinning strings in $A d S_{5} \times S^{5}$ : New integrable system relations," Phys. Rev. D 69 (2004) 086009, arXiv:hep-th/0311004.

[37] I. Y. Park, A. Tirziu and A. A. Tseytlin, "Spinning strings in AdS $S_{5} \times S^{5}$ : One-loop correction to energy in $\mathfrak{s l}(2)$ sector, "JHEP 0503 (2005) 013, arXiv: hep-th/0501203.

[38] S. Schafer-Nameki, M. Zamaklar and K. Zarembo, "How accurate is the quantum string Bethe ansatz?," JHEP 0612 (2006) 020, arXiv:hep-th/0610250.

[39] G. Arutyunov, S. Frolov and M. Zamaklar, "Finite-size effects from giant magnons," arXiv:hep-th/0606126. - K. Okamura and R. Suzuki, "A perspective on classical strings from complex sine-Gordon solitons," Phys. Rev. D 75, 046001 (2007), arXiv:hep-th/0609026. • D. Astolfi, V. Forini, G. Grignani and G. W. Semenoff, "Gauge invariant finite size spectrum of the giant magnon," arXiv:hep-th/0702043.

[40] D. M. Hofman and J. M. Maldacena, "Giant magnons," J. Phys. A 39 (2006) 13095, arXiv:hep-th/0604135.

[41] R. C. Brower, J. Polchinski, M. J. Strassler and C. I. Tan, "The pomeron and gauge / string duality," arXiv:hep-th/0603115.

[42] A. V. Belitsky, A. S. Gorsky and G. P. Korchemsky, "Logarithmic scaling in gauge / string correspondence," Nucl. Phys. B 748 (2006) 24 arXiv:hep-th/0601112.

[43] A. V. Kotikov and L. N. Lipatov, "On the highest transcendentality in $\mathcal{N}=4$ SUSY", Nucl. Phys. B 769 (2007) 217, arXiv: hep-th/0611204. • M. K. Benna, S. Benvenuti, 
I. R. Klebanov and A. Scardicchio, "A test of the AdS/CFT correspondence using highspin operators," arXiv:hep-th/0611135. • L. F. Alday, G. Arutyunov, M. K. Benna, B. Eden and I. R. Klebanov, "On the Strong Coupling Scaling Dimension of High Spin Operators," arXiv:hep-th/0702028. • I. Kostov, D. Serban and D. Volin, "Strong coupling limit of Bethe ansatz equations," arXiv:hep-th/0703031. - M. Beccaria, G. F. De Angelis and V. Forini, "The scaling function at strong coupling from the quantum string Bethe equations," arXiv:hep-th/0703131.

[44] S. S. Gubser, I. R. Klebanov and A. M. Polyakov, "A semi-classical limit of the gauge/string correspondence", Nucl. Phys. B 636 (2002) 99, arXiv:hep-th/0204051. - S. Frolov and A. A. Tseytlin, "Semiclassical quantization of rotating superstring in $A d S_{5} \times S^{5}$ ", JHEP 0206 (2002) 007, arXiv:hep-th/0204226.

[45] N. Dorey, D. M. Hofman and J. Maldacena, "On the singularities of the magnon S-matrix," arXiv:hep-th/0703104.

[46] J. Ambjorn, R. A. Janik and C. Kristjansen, "Wrapping interactions and a new source of corrections to the spin-chain / string duality," Nucl. Phys. B 736 (2006) 288, arXiv: hep-th/0510171.

[47] A. Rej, D. Serban and M. Staudacher, "Planar $\mathcal{N}=4$ gauge theory and the Hubbard model", JHEP 0603, 018 (2006), arXiv:hep-th/0512077. • G. Feverati, D. Fioravanti, P. Grinza and M. Rossi, "Hubbard's adventures in $N=4$ SYM-land? Some non-perturbative considerations on finite length operators," arXiv:hep-th/0611186. 